\title{
Multimodality management of soft tissue tumors in the extremity
}

\author{
Aimee M. Crago, M.D., Ph.D., FACS ${ }^{1,2}$ and Ann Y. Lee, M.D. ${ }^{1}$ \\ ${ }^{1}$ Sarcoma Disease Management Team, Department of Surgery, Memorial Sloan Kettering Cancer \\ Center \\ ${ }^{2}$ Department of Surgery, Weill Cornell Medical College
}

\begin{abstract}
Most extremity soft tissue sarcomas present as a painless mass. Workup should generally involve cross-sectional imaging with MRI, as well as a core biopsy for pathologic diagnosis. Limb-sparing surgery is the standard of care, and may be supplemented with radiation for histologic subtypes at higher risk for local recurrence and chemotherapy for those at higher risk for distant metastases. This article reviews the work-up and surgical approach to extremity soft tissue sarcomas, as well as the role for radiation and chemotherapy, with particular attention given to the distinguishing characteristics of some of the most common subtypes.
\end{abstract}

\section{Keywords}

Soft tissue sarcoma; extremity sarcoma; limbs; resection; radiotherapy

\section{Introduction}

\begin{abstract}
Soft tissue sarcoma (STS) is a term referring to approximately 100 different subtypes of cancer. ${ }^{1}$ These diseases are rare, and as a group are diagnosed in only approximately 12,000 patients in the United States each year. ${ }^{2}$ While STS can be identified in any site within the body, $40 \%$ are located in the extremities, and multimodality treatment is used to manage patients with localized disease. ${ }^{3}$ What combination of surgery, radiation and systemic treatment is best for a particular patient depends on histologic subtype, which is diagnosed using a combination of cross-sectional imaging, microscopy, and molecular diagnostics. The most common histologies in the extremity are liposarcoma, undifferentiated pleomorphic sarcoma (UPS), myxofibrosarcoma, and synovial sarcoma (Figure 1). Each carries a different risk for distant metastases and local recurrence; for example, DFSP carries a higher long-term risk of local recurrence than leiomyosarcoma, but its risk of metastasis is very
\end{abstract}

\footnotetext{
Corresponding author: Aimee M. Crago, MD, PhD, FACS, Gastric and Mixed Tumor Service, Department of Surgery, 1275 York Avenue, H1220, New York, NY 10065, (212) 639-4807, cragoa@ @skcc.org, Ann Y.Lee, M.D., Department of Surgery, 1275 York Avenue, H1220, New York, NY 10065, (212) 639-4807, leea5@mskcc.org.

Publisher's Disclaimer: This is a PDF file of an unedited manuscript that has been accepted for publication. As a service to our customers we are providing this early version of the manuscript. The manuscript will undergo copyediting, typesetting, and review of the resulting proof before it is published in its final citable form. Please note that during the production process errors may be discovered which could affect the content, and all legal disclaimers that apply to the journal pertain.

Disclosures: The authors have no disclosures to report.
} 
much lower. Below we present an algorithm for diagnosis and treatment of STS, highlighting modifications that should be made based on the biologic behavior of specific histologic subtypes.

\section{Clinical Presentation and Diagnosis}

Most patients eventually diagnosed with a STS present with a painless mass. Over $90 \%$ of painless masses are benign lesions such as lipomas. Therefore, sarcomas are sometimes initially diagnosed as lipomas, resulting in a delay in the correct diagnosis. In general, lipomas tend to:

$$
\begin{aligned}
& \text { - } \quad \text { be softer, } \\
& \text { - } \quad \text { be in a subcutaneous location, } \\
& \text { - } \quad \text { have a history of prolonged stability, }
\end{aligned}
$$

In contrast, STS may be firm, deep, enlarging over time, multifocal, and associated with neovascularization of the overlying skin. Radicular pain or swelling in the distal extremity related to underlying neurovascular involvement may reflect locally advanced disease. A history of nearby trauma may be reported by the patient, but it is unclear that trauma can initiate STS development. More likely, trauma brings attention to a preexisting mass.

\section{Imaging the primary tumor}

Work-up of an extremity mass begins with cross-sectional imaging for all but the smallest superficial lesions $(<2-3 \mathrm{~cm})$, which can be managed with an excisional biopsy. MRI with contrast is the preferred study modality, as it provides detailed anatomic information necessary for surgical planning. MRI allows the vascular structures to be easily delineated, and $\mathrm{T} 1$ images allow the clinician to trace branches of major peripheral nerves. Computed tomography (CT) is beneficial only when bony involvement is expected and complex reconstruction may be necessary during surgical resection of the tumor. The MRI appearance can also be predictive of histologic subtype (Figure 2). For example, myxoid lesions are associated with high $\mathrm{T} 2$ signal, even in the absence of gadolinium contrast. This is the case for myxofibrosarcoma, which often has enhancing tails extending from the multifocal, nodular components of the lesion, highlighting its infiltrative nature. ${ }^{4}$ Multifocal nodules can also be observed with myxofibrosarcoma. Collagenous regions are low in signal on both T1 and T2 imaging, so a persistent low signal in regions of the tumor may indicate a desmoid-type fibromatosis or a collagenous fibroma. However, in none of these instances is the diagnosis pathognomonic, and pre-treatment biopsy is recommended. There is only one STS type for which imaging is considered diagnostic (and only when reviewed by an experienced radiologist); this type is atypical lipomatous neoplasm/well-differentiated liposarcoma/dedifferentiated liposarcoma. This type has a signal profile almost identical to subcutaneous fat, but with enhancing septae and, if a dedifferentiated component is present, with solid nodules. ${ }^{5,6}$ 


\section{Biopsy techniques}

When imaging is not pathognomonic, biopsy is necessary before treatment is planned.

Historically, this was performed by incisional biopsy. However, subsequent studies have shown that core biopsy of STS is accurate not only in demonstrating the presence of malignancy, but also in determining grade (in $88 \%$ of cases) and histologic subtype (in $75 \%$ of cases). ${ }^{7}$ Core biopsy often provides enough tissue for immunohistochemistry and cytogenetic studies, which can be of significant benefit in diagnosis. Characteristic genomic alterations for common histologic subtypes of extremity sarcoma and their associated diagnostic tests are presented in Table 1. While fine needle aspiration has been examined as a means of biopsying soft tissue lesions at the time of presentation, its utility is limited by the rarity of specialists trained both in cytology and STS histology and the limited amount of tissue obtained from such specimens. Fine needle aspiration may be appropriate for initial diagnosis of malignant tumor or confirmation of a recurrence, but it is often inadequate for histologic subtyping. Regardless of the type of biopsy performed, care should be made to orient the incision or core needle in such a way that the biopsy tract can be completely excised at the time of eventual surgery. For incisional or excisional biopsy, this means orienting the incision longitudinally along the extremity. This greatly facilitates closure of the incision at the time of reexcision. If the incisional biopsy is oriented transversely, removing the incision with requisite margin during the definitive resection will often leave a defect requiring skin graft.

\section{Staging and extent of disease work-up}

AJCC staging (Table 2) takes into account tumor size and depth (T), nodal metastases (N), distant metastases (M), and histologic grade (G). Unlike carcinomas, STSs rarely metastasize to regional lymph nodes. The histologic subtypes with the highest rates of lymph node metastasis are angiosarcoma, epithelioid sarcoma, and embryonal rhabdomyosarcoma. The more common route of metastasis is direct hematogenous spread. Most STSs metastasize preferentially to the lung, and NCCN staging guidelines generally recommend chest X-ray or CT scan to rule out distant disease. In general, the use of chest imaging can be tailored to the metastatic risk associated with a given lesion. This risk can be estimated based on AJCC staging or, more accurately, based on prognostic nomograms, many of which are subtype-specific. ${ }^{8-10}$ For example, atypical lipomatous tumors do not metastasize. In general, low-grade STSs rarely spread; local recurrence is more common and staging can be completed with preoperative chest X-ray. Large, high-grade UPS or leiomyosarcoma have rates of disease-specific death that may approach 50\%, and distant metastases are common, so chest CT would be reasonable as a means of determining extent of disease for these high-risk patients. Special consideration is given to myxoid/round cell liposarcoma, which has a unique pattern of spread with a propensity for metastasis to soft tissue fat pads and the spine. CT of the chest, abdomen and pelvis is generally performed in high-risk patients, and MRI of the total spine can be considered. ${ }^{11,12}$ PET scan is not clearly warranted for routine extent of disease work-up in STS; many histologic subtypes are not FDG-sensitive, and PET is less sensitive than CT for identifying subcentimeter pulmonary nodules. 


\section{Surgical Approach}

Benign soft tissue tumors can often be observed, and tumors of a few types, such as nodular fasciitis, may spontaneously regress. However, for intermediate and malignant subtypes of soft tissue tumors, surgery has been considered the 'gold-standard' of treatment. Historically, STS of the extremity was treated with amputation. However, in a randomized clinical trial of 43 patients who received adjuvant chemotherapy and underwent limb-sparing surgery followed by radiation or amputation (2:1 randomization), only $15 \%$ of patients undergoing limb-sparing experienced local recurrences, and the two treatment groups did not differ in five year disease-specific or overall survival. ${ }^{13}$ For this reason, limb-sparing procedures are now the standard for treatment of extremity STS. Initially, this was performed by resecting the entire involved muscle, but the current standard for most STS histologies is resection of a 1-cm margin. For superficial lesions, the underlying muscular fascia is removed with the specimen. Similarly, for intramuscular tumors, the fascial barrier between muscle bodies or compartments can provide an adequate barrier and should be resected with the specimen. The key principles of surgery for extremity sarcomas are summarized in Table 3.

In the extremity, extent of resection can sometimes be limited by adjacent neurovascular bundles. Generally, the STS is resected away from a neurovascular bundle with the overlying fascial layer (e.g., femoral sheath) or the perineurium so as to optimize margins. Encasement of a major neurovascular structure by a low-grade STS is generally managed by bivalving the tumor to minimize morbidity. Encasement by a high-grade lesion may necessitate resection of a major neurovascular structure. Arterial reconstruction can be planned when the artery is involved; venous reconstruction is generally unsuccessful and is often deferred. If the patient develops venous congestion, compression and elevation are prescribed to minimize morbidity as alternative routes of venous drainage develop.

Patients should be carefully counseled regarding expected results of a nerve resection. Sciatic or peroneal resection is generally well tolerated, but results in foot drop and requires ankle bracing. Interruption of the tibial branches of the sciatic causes paresthesias on the plantar aspect of the foot, and patients should be instructed to monitor their feet routinely for trauma that may occur secondary to an insensate foot. ${ }^{14}$ Femoral nerve injury results in instability of the knee and may necessitate bracing, particularly in older patients, and patients are at increased risk of fracture in the long term. ${ }^{15}$ Resection of one of the three major nerves in the upper extremity will generally allow some retention of function, but may necessitate bracing of the limb, result in significant paresthesias, or limit opposition. Advanced maneuvers such as tendon transfers can be considered in collaboration with a hand specialist.

Special consideration should be given to tumors associated with high rates of local recurrence, mainly myxofibrosarcoma, dermatofibrosarcoma protuberans (DFSP), and desmoid-type fibromatosis. ${ }^{16-18}$ Both myxofibrosarcoma and DFSP have microscopic components that extend outward from the visible tumor. In the context of myxofibrosarcoma, these may be visible on MRI as enhancing tails (figure 2A), and 2-cm margins should be planned circumferentially around the dominant nodules and these tails. In the context of DFSP, a reasonable margin is $2 \mathrm{~cm}$ around visible disease. In a retrospective 
analysis of 206 DFSPs, approximately $85 \%$ of surgeries planned with 1-2 cm margins resulted in complete microscopic resection. ${ }^{19}$ Both DFSP with fibrosarcomatous degeneration and myxofibrosarcoma may invade through fascial margins, so these planes should not be considered as adequate alternatives to the full width of the margin as they can be in most tumor histologies.

Desmoid-type fibromatosis was historically treated like a low-grade fibrosarcoma and managed with aggressive surgical extirpation. However, surgery is currently being used less aggressively for all but the smallest extremity desmoids that can be removed with wide margins, for three reasons. First, related metastases have never been identified for desmoids. Second, extremity desmoids have high rates of local recurrence (>50-60\% in some series), ${ }^{20-22}$ and repeated surgeries or amputation cause significant morbidity. Finally, over time, many desmoids remain stable in size or regress. ${ }^{23}$ Observation is a reasonable alternative, or in the context of symptoms, systemic therapy can be considered. Traditional agents (i.e. doxorubicin or vincristine/methotrexate combinations) and targeted therapies (i.e. sorafenib and notch inhibitors) show responses and alleviate symptoms in clinical trials, so they are being used increasingly in management of patients with high-risk desmoids. ${ }^{24-26}$ Aggressive surgery is less and less frequently considered for some other histologic subtypes for which, as for desmoids, rates of local or regional recurrence are high even after complete microscopic resection, progression follows an indolent course, and surgery is associated with significant morbidity. These include multifocal epithelioid hemangioendothelioma in a single extremity and tenosynovial giant cell tumors, which can be infiltrative lesions affecting entire muscle compartments and may be responsive to tyrosine kinase inhibitors.

Lymph node metastases occur in less than five percent of soft tissue sarcomas so that routine sentinel lymph node biopsy or nodal dissection is not performed. When clinically positive nodes are present, prognosis is poor, however. In these instances, radical lymphadenectomy for isolated regional nodal recurrence is associated with improved survival. The histologic subtypes most frequently associated with lymph node metastases are angiosarcoma, rhabdomyosarcoma, clear cell, and epithelioid sarcoma where rates of nodal metastases are approximately $25 \% .{ }^{27-29}$ In these histologies, it has been proposed that sentinel lymph node biopsy may be of prognostic or therapeutic benefit. However, the role of sentinel lymph node biopsy is unclear as prospective studies have only demonstrated a 5-7\% rate of occult lymph node metastases in patients with these higher risk subtypes and no survival advantage has been observed in patients undergoing the procedure. ${ }^{30,31}$ The small number of patients in these studies makes it difficult to draw any definitive conclusions, but the majority of benefit obtained from sentinel node biopsy is likely related to improved prognostication as opposed to true therapeutic benefit, and we do not routinely recommend the procedure even in high risk histologies.

\section{Clinical Outcomes}

Rates of local recurrence and distant recurrence differ by histologic subtype. For extremity STS, distant metastases are the primary cause of sarcoma-specific death. This is in contrast to retroperitoneal sarcomas, where local recurrence can cause significant morbidity and even disease-specific death. The local recurrence-free survival and distant recurrence-free 
survival of the 6 most common subtypes of extremity STS are shown in Figure 3.

Highlighted by these survival curves is the high rate of local recurrence for myxofibrosarcoma, high rate of early distant recurrence of undifferentiated pleomorphic sarcoma, and particularly low rate of distant recurrence for DFSP. Even within the group of liposarcomas there are differences, with pleomorphic liposarcomas and round cell liposarcomas having a notably higher risk of distant recurrence. These differences in local and distant recurrence help inform patient management and follow-up. General risk factors for sarcoma-related death are as follows:

$\begin{array}{ll}\text { - } & \text { size }>5 \mathrm{~cm}, \\ \text { - } & \text { age }>50 \text { years, } \\ \text { - } & \text { deep location, } \\ \text { - } & \text { high grade, } \\ & \text { incomplete gross resection. }\end{array}$

\section{Multimodality Treatment}

Adjuvant radiation and chemotherapy are considered in conjunction with surgery in an attempt to prevent local or distant recurrence. While adjuvant and neoadjuvant radiation have relatively clear indications, the role of adjuvant chemotherapy is controversial, with wide differences in treatment strategies employed even across highly-specialized sarcoma centers.

\section{Adjuvant and neoadjuvant radiation}

The role of local radiation to prevent local recurrence has been defined in a range of clinical trials and prospective studies. Adjuvant radiation was used in conjunction with limb-sparing surgery in the NIH-led trial defining amputation as unnecessary in routine management of STS. A second randomized trial, again led by investigators at the NIH, examined whether adjuvant radiation affects patient outcomes. Ninety-one patients were randomized to undergo adjuvant radiation vs. observation after their surgery. Local recurrence rates were lower in patients receiving adjuvant radiation than in those on observation, overall survival did not significantly differ. Similar findings were recorded in subsets of patients with lowgrade and high-grade tumors, and these results were durable when reanalyzed with a median follow-up of 18 years. ${ }^{32,33}$ Similar findings were observed when patients were randomized to receive brachytherapy vs. no further therapy after limb-sparing surgery; specifically, lower rates of local recurrence were observed in patients receiving radiation than in those treated with surgery alone. In this context, however, the observed benefit was restricted to patients with high-grade lesions. Again, radiation was not associated with better overall or diseasespecific survival. ${ }^{34}$

Because adjuvant radiation has not been shown to improve rates of overall survival, the risks associated with radiation should be considered prior to recommending the treatment for a given patient. Radiation can result in post-operative wound complications, radiationassociated fracture, fibrosis in nearby joints, neuritis, and secondary sarcomas. For this 
reason, when the baseline risk of local recurrence is small or a local recurrence could be easily salvaged with secondary surgeries, adjuvant radiation is not advised. A prospective study of patients with T1 STS treated with surgery alone showed that forgoing radiation appeared to be safe in most cases. At a median follow-up of 75 months, 5-year local recurrence rates after R0 resection $(\mathrm{n}=74)$ were only $8 \% .{ }^{35} \mathrm{In}$ low-risk lesions such as these, most clinicians would defer the use of radiation unless local recurrence would be salvageable only with a morbid procedure. Previous studies have identified age greater than $50^{36,37}$, microscopically positive margins ${ }^{36-41}$, high grade ${ }^{39-42}$, deep location 37,40 , and recurrent tumors ${ }^{36,37}$ as risk factors for local recurrence. Many of these factors, as well as atypical lipomatous tumor histology (a positive prognostic factor), have been integrated into a nomogram that estimates a patient's risk of local recurrence after surgery alone and can therefore be used to predict whether the patient is likely to benefit from adjuvant treatment. ${ }^{41}$

Radiation can be administered in either the adjuvant or the neoadjuvant setting; to determine which is appropriate for the individual patient, the clinician should carefully consider the risks and benefits of each regimen (Table 4). These risks and benefits were defined in randomized trial of 94 patients reported by O'Sullivan et al. Participants received either 50 Gy preoperatively with a 5-Gy postoperative boost to the tumor bed or $66 \mathrm{~Gy}$ administered post-operatively. At a median follow-up of 3.3 years, the two groups had equivalent rates of local recurrence. However, significant wound complications (defined as requiring operative intervention, prolonged packing, or invasive procedures to minimize complications) were more frequent in those who received neoadjuvant radiation than in those who received their treatment in the adjuvant setting ( $35 \%$ vs. $17 \%$; $\mathrm{p}=0.01$ ). This difference was exclusively related to high rates of wound complications in the thigh (affecting $45 \%$ of patients with tumors resected from the upper leg). ${ }^{43}$

Despite its association with wound complications in the thigh, neoadjuvant radiation can have significant benefit compared to that adjuvant radiation. Standard delivery of radiation involves radiating a field that extends $1.5 \mathrm{~cm}$ radially and $4 \mathrm{~cm}$ proximally and distally from the site of the tumor or surgical bed. ${ }^{43}$ Because the tumor is resected with a margin of normal tissue where possible, a larger volume of normal tissue must be included in the radiation field if radiation is given to the surgical bed as opposed to the tumor in situ. In addition, adjuvant radiation is generally given to doses of $66 \mathrm{~Gy}$ as opposed to preoperative doses that are maximally $55 \mathrm{~Gy}$. Neoadjuvant radiation is, therefore, theoretically able to minimize risks of radiation-associated side effects such as neuritis. Long-term follow-up of patients in the O'Sullivan et al. study has demonstrated concrete reductions in joint fibrosis, which was noted in $31 \%$ of patients who received neoadjuvant radiation and $48 \%$ of patients who received adjuvant radiation ( $\mathrm{p}=0.07$ ). In addition, patients receiving neoadjuvant treatment tended to have less edema (15\% vs. $23 \%)$ and less joint stiffness (18\% vs. $23 \%) .{ }^{44}$ Implementation of newer modes of radiation delivery such as image-guided intensity modulated radiotherapy (IMRT) or proton beam therapy may minimize non-specific injury to normal margins and minimize side effects further. IMRT is similar to conventional radiation therapy for minimizing local recurrence, and in an initial IMRT series postoperative edema was $11 \%$ and joint fibrosis was $5.6 \%$, frequencies lower than in historical controls. No joint fractures were reported. ${ }^{45}$ 
Given the increased availability of modalities such as IMRT and proton beam therapy, most institutions now routinely use neoadjuvant radiation therapy for patients at high risk of local recurrence. In our own case, due to the persistent increase in wound complications related to neoadjuvant treatment and the increased need for complex wound closures that may complicate re-resection in the event of local recurrence, we prescribe neoadjuvant radiation selectively. It is prescribed for tumors near a joint, preferentially in the upper extremity and in instances where locally advanced disease may necessitate extensive resection. For tumors in the upper thigh, where wound complications are highest, we tend to resect first and treat with radiation in the adjuvant setting. Radiation should not be prescribed in the neoadjuvant setting if preoperative biopsy is inconclusive regarding grade and/or histology, as radiation would constitute overtreatment if the tumor is low risk or benign.

\section{Adjuvant and neoadjuvant chemotherapy}

Recommendations regarding the prescription of adjuvant chemotherapy in patients with STS of the extremity vary greatly, even among high-volume specialty centers. This variability comes from the range of results in randomized clinical trials and the limitations associated with these trials in general. Early trials examined small cohorts of patients undergoing surgery (with or without radiation), who were randomized to undergo adjuvant systemic treatment vs. observation. In an early study, 88 patients with FNCLCC grade II or III STS were randomized to systemic therapy (epirubicin with or without ifosfamide) vs. no systemic therapy. While the systemic therapy group had significantly better recurrence-free survival ( $44 \%$ vs. $69 \%$ at 5 years; $\mathrm{p}=0.01$ ), no difference in overall survival was identified. ${ }^{46}$ Several subsequent studies, generally randomizing patients to observation versus doxorubicin/ifosfamide-based regimens, had similar outcomes with no consistent evidence that systemic regimens improve overall or disease-specific survival.

The most recent of these randomized trial reports was an EORTC-sponsored trial (EORTC 62931). ${ }^{36}$ Patients with grade II or III STS $(\mathrm{n}=351)$ were randomized to either five cycles of doxorubicin and ifosfamide or to no chemotherapy following treatment for local disease. In this trial, no difference was observed between the two groups in either relapse-free survival or overall survival. As part of the study, however, the investigators performed a metaanalysis of data presented in previously reported clinical trials as well as their own data for a combined 1071 patients receiving adjuvant chemotherapy and 1074 observed following treatment for local disease. While $46.5 \%$ of patients who did not receive adjuvant chemotherapy died during follow-up, only $41.4 \%$ of patients in adjuvant arms died (hazard ratio $0.86, \mathrm{p}=0.02$ ), suggesting an absolute risk reduction of $\sim 5 \%$ associated with adjuvant chemotherapy. While the paucity of survival benefit has led many groups to argue against the routine use of adjuvant chemotherapy, the meta-analysis result, combined with the limitations of published trials (specifically the heterogeneity of tumor types eligible for entrance), has led others to argue that at least a subset of patients may have more substantial treatment benefit from treatment than that observed in the cohort as a whole. ${ }^{47}$

The argument in favor of chemotherapy for subsets of sarcoma patients has been further bolstered by subset analysis of the patient cohort enrolled on EORTC 62931. In ad hoc analyses, the patients whose outcomes tended to be most improved in the adjuvant 
chemotherapy arm were those patients with a tumors that were grade III, located in the limb (versus trunk or central site), and greater than or equal to $10 \mathrm{~cm}$ in diameter. These subset analyses did not reach statistical significance, but results leave open the possibility that these clinical characteristics may define cohorts who would receive the most benefit from adjuvant chemotherapy. ${ }^{47}$ Retrospective series have led further credence to this hypothesis, particularly those examining patients with single histologic subtype of STS with known sensitivity to chemotherapy. An example is a review of 255 patients treated for localized synovial sarcoma. Data for patients who did not receive adjuvant chemotherapy was used to contract a nomogram based on tumor size and site, among other variables. Patients treated with adjuvant chemotherapy, however, had significantly better 3-year disease-specific survival than predicted by the nomogram. ${ }^{9}$ Another study suggested that improved outcomes in patients with pleomorphic or round cell liposarcoma are associated with receipt of adjuvant ifosfamide-based chemotherapy. ${ }^{48}$

In general, our own practice has been to consider neoadjuvant chemotherapy for high-risk patients. The neoadjuvant setting allows for observation of the tumor in situ and for early discontinuation of the treatment if there is no evidence of response. An additional criterion for chemotherapy is the relative chemosensitivity of the STS subtype (Table 5). For example, rhabdomyosarcoma and Ewing sarcoma patients have risks of sarcoma-specific death that can be over 50\%, and these tumors are sensitive to chemotherapy; therefore, in our practice, all of these patients receive neoadjuvant systemic therapy. Moderately chemosensitive histologies are treated with chemotherapy when a high-risk tumor is over $5 \mathrm{~cm}$ in size (e.g., synovial sarcoma), a moderate-risk tumor is over $8 \mathrm{~cm}$ (e.g., undifferentiated pleomorphic sarcoma), and a low-risk tumor is over $10 \mathrm{~cm}$ (e.g., myxofibrosarcoma). Chemoresistant histologies such as dedifferentiated liposarcoma are not treated with neoadjuvant chemotherapy.

\section{Isolated limb perfusion}

Limb-sparing surgery is the standard of care for extremity sarcoma; however, resection of some extremity sarcomas requires an amputation due to involvement of major neurovascular bundles or multifocality. Isolated limb perfusion (ILP) has been used in patients that would otherwise require an amputation in an attempt to convert them to a limb-sparing operation. ILP with melphalan alone had limited success, ${ }^{49}$ however combination treatment with tumor necrosis factor-a (TNFa) has been shown to have clinical response and limb salvage rates of $70-80 \% .{ }^{50-55}$ This finding has not significantly altered therapeutic paradigms in the US, however, as TNFa is not available in North American centers.

\section{Follow-up}

After resection and adjuvant therapy for localized STS, the NCCN guidelines generally recommend follow-up every 3-6 months for 2-3 years and every 6-12 months thereafter, depending on stage. Patients with tumors of higher metastatic risk generally undergo chest $\mathrm{X}$-ray or chest CT. In practice, low-grade tumors and small, high-grade tumors may have minimal risk of distant metastases and are initially followed every 6-12 months. More frequent surveillance is undertaken for patients with large, high-grade tumors identified in 
the deep compartments of the extremity. Care should be taken for certain histologies to ensure that screening exams are tailored to the unique patterns of spread. For example, round cell liposarcoma has a propensity to metastasize to fat pads, so after resection, CT of the abdomen and pelvis may be considered in addition to chest scans. Local recurrence can generally be detected by physical exam, though MRI is considered for deep lesions and those with infiltrative histologies such as myxofibrosarcoma that may not be palpable early.

\section{Summary}

Workup of a mass suspicious for a soft tissue sarcoma starts with a detailed history and physical, followed by cross-sectional imaging (MRI preferred) and a well-planned core needle biopsy for pathologic diagnosis. Rates of local recurrence, distant metastasis (typically to lungs), and sarcoma-specific survival vary significantly among different histologic subtypes, and these differences help inform multimodality treatment planning.

The standard of care for extremity sarcoma is limb-sparing surgery with a margin of 1-2 cm (depending on histology), with fascial boundaries providing an acceptable margin to limit morbidity of the resection when major neurovascular or bony structures are in close proximity. Radiation therapy should be considered for tumors with a high risk of local recurrence. The timing of radiation is best determined by weighing the increased risk of wound complications from neoadjuvant radiation against the increased risk of side effects to surrounding tissues and joints from adjuvant radiation. The use of adjuvant chemotherapy is controversial; however, there are relative indications for neoadjuvant chemotherapy for chemosensitive subtypes with moderate or high risk of distant metastases. Currently there are approximately 100 histologic subtypes of soft tissue sarcoma with variable biology, and these nuances in the therapeutic algorithm highlight the importance of patients being evaluated and managed by a multidisciplinary team with experience and expertise in sarcoma.

\section{Acknowledgments}

This work was supported by the Memorial Sloan Kettering Cancer Center Core Grant (P30 CA008748) and the Kristen Ann Carr Fund. The authors thank Janet Novak for editorial assistance.

\section{Key Abbreviations Box}

$\begin{array}{ll}\text { AJCC } & \text { American Joint Committee on Cancer } \\ \text { DFSP } & \text { dermatofibrosarcoma protuberans } \\ \text { IMRT } & \text { intensity-modulated radiotherapy } \\ \text { NCCN } & \text { National Comprehensive Cancer Network } \\ \text { STS } & \text { soft tissue sarcoma } \\ \text { UPS } & \text { undifferentiated pleomorphic sarcoma }\end{array}$




\section{References}

1. WHO Classification of Tumours of Soft Tissue and Bone. 4th. Lyon, France: IARC; 2013.

2. Siegel RL, Miller KD, Jemal A. Cancer statistics, 2015. CA Cancer J Clin. 2015 Jan; 65(1):5-29. [PubMed: 25559415]

3. Brennan MF, Antonescu CR, Moraco N, Singer S. Lessons learned from the study of 10,000 patients with soft tissue sarcoma. Ann Surg. 2014 Sep; 260(3):416-421. discussion 421-412. [PubMed: 25115417]

4. Lefkowitz RA, Landa J, Hwang S, et al. Myxofibrosarcoma: prevalence and diagnostic value of the "tail sign" on magnetic resonance imaging. Skeletal radiology. 2013 Jun; 42(6):809-818. [PubMed: 23318907]

5. Jelinek JS, Kransdorf MJ, Shmookler BM, Aboulafia AJ, Malawer MM. Liposarcoma of the extremities: MR and CT findings in the histologic subtypes. Radiology. 1993 Feb; 186(2):455-459. [PubMed: 8421750]

6. Murphey MD, Arcara LK, Fanburg-Smith J. From the archives of the AFIP: imaging of musculoskeletal liposarcoma with radiologic-pathologic correlation. Radiographics. 2005 Sep-Oct; 25(5):1371-1395. [PubMed: 16160117]

7. Heslin MJ, Lewis JJ, Woodruff JM, Brennan MF. Core needle biopsy for diagnosis of extremity soft tissue sarcoma. Ann Surg Oncol. 1997 Jul-Aug;4(5):425-431. [PubMed: 9259971]

8. Eilber FC, Brennan MF, Eilber FR, Dry SM, Singer S, Kattan MW. Validation of the postoperative nomogram for 12-year sarcoma-specific mortality. Cancer. 2004 Nov 15; 101(10):2270-2275. [PubMed: 15484214]

9. Canter RJ, Qin LX, Maki RG, Brennan MF, Ladanyi M, Singer S. A synovial sarcoma-specific preoperative nomogram supports a survival benefit to ifosfamide-based chemotherapy and improves risk stratification for patients. Clin Cancer Res. 2008 Dec 15; 14(24):8191-8197. [PubMed: 19088035]

10. Dalal KM, Kattan MW, Antonescu CR, Brennan MF, Singer S. Subtype specific prognostic nomogram for patients with primary liposarcoma of the retroperitoneum, extremity, or trunk. Ann Surg. 2006 Sep; 244(3):381-391. [PubMed: 16926564]

11. Hoffman A, Ghadimi MP, Demicco EG, et al. Localized and metastatic myxoid/round cell liposarcoma: clinical and molecular observations. Cancer. 2013 May 15; 119(10):1868-1877. [PubMed: 23401071]

12. Schwab JH, Boland PJ, Antonescu C, Bilsky MH, Healey JH. Spinal metastases from myxoid liposarcoma warrant screening with magnetic resonance imaging. Cancer. 2007 Oct 15; 110(8): 1815-1822. [PubMed: 17724681]

13. Rosenberg SA, Tepper J, Glatstein E, et al. The treatment of soft-tissue sarcomas of the extremities: prospective randomized evaluations of (1) limb-sparing surgery plus radiation therapy compared with amputation and (2) the role of adjuvant chemotherapy. Ann Surg. 1982 Sep; 196(3):305-315. [PubMed: 7114936]

14. Brooks AD, Gold JS, Graham D, et al. Resection of the sciatic, peroneal, or tibial nerves: assessment of functional status. Ann Surg Oncol. 2002 Jan-Feb;9(1):41-47. [PubMed: 11829429]

15. Jones KB, Ferguson PC, Deheshi B, et al. Complete femoral nerve resection with soft tissue sarcoma: functional outcomes. Ann Surg Oncol. 2010 Feb; 17(2):401-406. [PubMed: 19841985]

16. Mentzel T, Calonje E, Wadden C, et al. Myxofibrosarcoma. Clinicopathologic analysis of 75 cases with emphasis on the low-grade variant. Am J Surg Pathol. 1996 Apr; 20(4):391-405. [PubMed: 8604805]

17. Huang HY, Lal P, Qin J, Brennan MF, Antonescu CR. Low-grade myxofibrosarcoma: a clinicopathologic analysis of 49 cases treated at a single institution with simultaneous assessment of the efficacy of 3-tier and 4-tier grading systems. Hum Pathol. 2004 May; 35(5):612-621. [PubMed: 15138937]

18. Fields RC, Hameed M, Qin LX, et al. Dermatofibrosarcoma protuberans (DFSP): predictors of recurrence and the use of systemic therapy. Ann Surg Oncol. 2011 Feb; 18(2):328-336. [PubMed: 20844969] 
19. Farma JM, Ammori JB, Zager JS, et al. Dermatofibrosarcoma protuberans: how wide should we resect? Ann Surg Oncol. 2010 Aug; 17(8):2112-2118. [PubMed: 20354798]

20. Crago AM, Denton B, Salas $S$, et al. A prognostic nomogram for prediction of recurrence in desmoid fibromatosis. Ann Surg. 2013 Aug; 258(2):347-353. [PubMed: 23532110]

21. Merchant NB, Lewis JJ, Woodruff JM, Leung DH, Brennan MF. Extremity and trunk desmoid tumors: a multifactorial analysis of outcome. Cancer. 1999 Nov 15; 86(10):2045-2052. [PubMed: 10570430]

22. Salas S, Dufresne A, Bui B, et al. Prognostic factors influencing progression-free survival determined from a series of sporadic desmoid tumors: a wait-and-see policy according to tumor presentation. J Clin Oncol. 2011 Sep 10; 29(26):3553-3558. [PubMed: 21844500]

23. Fiore M, Rimareix F, Mariani L, et al. Desmoid-type fibromatosis: a front-line conservative approach to select patients for surgical treatment. Ann Surg Oncol. 2009 Sep; 16(9):2587-2593. [PubMed: 19568815]

24. de Camargo VP, Keohan ML, D'Adamo DR, et al. Clinical outcomes of systemic therapy for patients with deep fibromatosis (desmoid tumor). Cancer. 2010 May 1; 116(9):2258-2265. [PubMed: 20187095]

25. Gounder MM, Lefkowitz RA, Keohan ML, et al. Activity of Sorafenib against Desmoid Tumor/ Deep Fibromatosis. Clin Cancer Res. 2011 Jun 15; 17(12):4082-4090. [PubMed: 21447727]

26. Messersmith WA, Shapiro GI, Cleary JM, et al. A Phase I, dose-finding study in patients with advanced solid malignancies of the oral gamma-secretase inhibitor PF-03084014. Clin Cancer Res. 2015 Jan 1; 21(1):60-67. [PubMed: 25231399]

27. Fong Y, Coit DG, Woodruff JM, Brennan MF. Lymph node metastasis from soft tissue sarcoma in adults. Analysis of data from a prospective database of 1772 sarcoma patients. Ann Surg. 1993 Jan; 217(1):72-77. [PubMed: 8424704]

28. Riad S, Griffin AM, Liberman B, et al. Lymph node metastasis in soft tissue sarcoma in an extremity. Clinical orthopaedics and related research. 2004 Sep.(426):129-134. [PubMed: 15346063]

29. Behranwala KA, A'Hern R, Omar AM, Thomas JM. Prognosis of lymph node metastasis in soft tissue sarcoma. Annals of surgical oncology. 2004 Jul; 11(7):714-719. [PubMed: 15231526]

30. Maduekwe UN, Hornicek FJ, Springfield DS, et al. Role of sentinel lymph node biopsy in the staging of synovial, epithelioid, and clear cell sarcomas. Ann Surg Oncol. 2009 May; 16(5):13561363. [PubMed: 19259743]

31. Andreou D, Boldt H, Werner M, Hamann C, Pink D, Tunn PU. Sentinel node biopsy in soft tissue sarcoma subtypes with a high propensity for regional lymphatic spread--results of a large prospective trial. Annals of oncology : official journal of the European Society for Medical Oncology / ESMO. 2013 May; 24(5):1400-1405. [PubMed: 23372051]

32. Yang JC, Chang AE, Baker AR, et al. Randomized prospective study of the benefit of adjuvant radiation therapy in the treatment of soft tissue sarcomas of the extremity. J Clin Oncol. 1998 Jan; 16(1):197-203. [PubMed: 9440743]

33. Beane JD, Yang JC, White D, Steinberg SM, Rosenberg SA, Rudloff U. Efficacy of adjuvant radiation therapy in the treatment of soft tissue sarcoma of the extremity: 20-year follow-up of a randomized prospective trial. Ann Surg Oncol. 2014 Aug; 21(8):2484-2489. [PubMed: 24756814]

34. Pisters PW, Harrison LB, Leung DH, Woodruff JM, Casper ES, Brennan MF. Long-term results of a prospective randomized trial of adjuvant brachytherapy in soft tissue sarcoma. J Clin Oncol. 1996 Mar; 14(3):859-868. [PubMed: 8622034]

35. Pisters PW, Pollock RE, Lewis VO, et al. Long-term results of prospective trial of surgery alone with selective use of radiation for patients with $\mathrm{T} 1$ extremity and trunk soft tissue sarcomas. Annals of surgery. 2007 Oct; 246(4):675-681. discussion 681-672. [PubMed: 17893504]

36. Pisters PW, Leung DH, Woodruff J, Shi W, Brennan MF. Analysis of prognostic factors in 1,041 patients with localized soft tissue sarcomas of the extremities. Journal of clinical oncology : official journal of the American Society of Clinical Oncology. 1996 May; 14(5):1679-1689. [PubMed: 8622088]

37. Gronchi A, Casali PG, Mariani L, et al. Status of surgical margins and prognosis in adult soft tissue sarcomas of the extremities: a series of patients treated at a single institution. Journal of clinical 
oncology : official journal of the American Society of Clinical Oncology. 2005 Jan 1; 23(1):96104. [PubMed: 15625364]

38. Stojadinovic A, Leung DH, Hoos A, Jaques DP, Lewis JJ, Brennan MF. Analysis of the prognostic significance of microscopic margins in 2,084 localized primary adult soft tissue sarcomas. Annals of surgery. 2002 Mar; 235(3):424-434. [PubMed: 11882765]

39. Trovik CS, Bauer HC, Alvegard TA, et al. Surgical margins, local recurrence and metastasis in soft tissue sarcomas: 559 surgically-treated patients from the Scandinavian Sarcoma Group Register. European journal of Cancer. 2000 Apr; 36(6):710-716. [PubMed: 10762742]

40. Coindre JM, Terrier P, Bui NB, et al. Prognostic factors in adult patients with locally controlled soft tissue sarcoma. A study of 546 patients from the French Federation of Cancer Centers Sarcoma Group. Journal of clinical oncology : official journal of the American Society of Clinical Oncology. 1996 Mar; 14(3):869-877. [PubMed: 8622035]

41. Cahlon O, Brennan MF, Jia X, Qin LX, Singer S, Alektiar KM. A postoperative nomogram for local recurrence risk in extremity soft tissue sarcomas after limb-sparing surgery without adjuvant radiation. Ann Surg. 2012 Feb; 255(2):343-347. [PubMed: 22143203]

42. Fabrizio PL, Stafford SL, Pritchard DJ. Extremity soft-tissue sarcomas selectively treated with surgery alone. International journal of radiation oncology, biology, physics. 2000 Aug 1; 48(1): 227-232.

43. O'Sullivan B, Davis AM, Turcotte R, et al. Preoperative versus postoperative radiotherapy in softtissue sarcoma of the limbs: a randomised trial. Lancet. 2002 Jun 29; 359(9325):2235-2241. [PubMed: 12103287]

44. Davis AM, O'Sullivan B, Turcotte R, et al. Late radiation morbidity following randomization to preoperative versus postoperative radiotherapy in extremity soft tissue sarcoma. Radiother Oncol. 2005 Apr; 75(1):48-53. [PubMed: 15948265]

45. O'Sullivan B, Griffin AM, Dickie CI, et al. Phase 2 study of preoperative image-guided intensitymodulated radiation therapy to reduce wound and combined modality morbidities in lower extremity soft tissue sarcoma. Cancer. 2013 May 15; 119(10):1878-1884. [PubMed: 23423841]

46. Petrioli R, Coratti A, Correale P, et al. Adjuvant epirubicin with or without Ifosfamide for adult soft-tissue sarcoma. American journal of clinical Oncology. 2002 Oct; 25(5):468-473. [PubMed: 12393986]

47. Woll PJ, Reichardt P, Le Cesne A, et al. Adjuvant chemotherapy with doxorubicin, ifosfamide, and lenograstim for resected soft-tissue sarcoma (EORTC 62931): a multicentre randomised controlled trial. The lancet Oncology. 2012 Oct; 13(10):1045-1054. [PubMed: 22954508]

48. Eilber FC, Eilber FR, Eckardt J, et al. The impact of chemotherapy on the survival of patients with high-grade primary extremity liposarcoma. Ann Surg. 2004 Oct; 240(4):686-695. discussion 695-687. [PubMed: 15383796]

49. Krementz ET, Carter RD, Sutherland CM, Hutton I. Chemotherapy of sarcomas of the limbs by regional perfusion. Annals of surgery. 1977 May; 185(5):555-564. [PubMed: 266397]

50. Eggermont AM, Schraffordt Koops H, Lienard D, et al. Isolated limb perfusion with high-dose tumor necrosis factor-alpha in combination with interferon-gamma and melphalan for nonresectable extremity soft tissue sarcomas: a multicenter trial. Journal of clinical oncology : official journal of the American Society of Clinical Oncology. 1996 Oct; 14(10):2653-2665. [PubMed: 8874324]

51. Eggermont AM, Schraffordt Koops H, Klausner JM, et al. Isolated limb perfusion with tumor necrosis factor and melphalan for limb salvage in 186 patients with locally advanced soft tissue extremity sarcomas. The cumulative multicenter European experience. Annals of surgery. 1996 Dec; 224(6):756-764. discussion 764-755. [PubMed: 8968230]

52. Deroose JP, Eggermont AM, van Geel AN, et al. Long-term results of tumor necrosis factor alphaand melphalan-based isolated limb perfusion in locally advanced extremity soft tissue sarcomas. Journal of clinical oncology : official journal of the American Society of Clinical Oncology. 2011 Oct 20; 29(30):4036-4044. [PubMed: 21931039]

53. Rastrelli M, Campana LG, Valpione S, Tropea S, Zanon A, Rossi CR. Hyperthermic isolated limb perfusion in locally advanced limb soft tissue sarcoma: A 24-year single-centre experience. 
International journal of hyperthermia : the official journal of European Society for Hyperthermic Oncology, North American Hyperthermia Group. 2016 Mar; 32(2):165-172.

54. Bhangu A, Broom L, Nepogodiev D, Gourevitch D, Desai A. Outcomes of isolated limb perfusion in the treatment of extremity soft tissue sarcoma: a systematic review. European journal of surgical oncology : the journal of the European Society of Surgical Oncology and the British Association of Surgical Oncology. 2013 Apr; 39(4):311-319.

55. Bonvalot $\mathrm{S}$, Laplanche A, Lejeune F, et al. Limb salvage with isolated perfusion for soft tissue sarcoma: could less TNF-alpha be better? Annals of oncology : official journal of the European Society for Medical Oncology / ESMO. 2005 Jul; 16(7):1061-1068. [PubMed: 15930042] 


\section{Key Points}

- Workup for an extremity mass suspicious for a soft tissue sarcoma includes cross-sectional imaging with an MRI and a core biopsy done in line with the planned incision.

- $\quad$ Most soft tissue sarcomas preferentially metastasize to the lungs. Therefore, staging should include a chest X-ray (for low-risk patients) or chest CT scan (for higher-risk patients).

- $\quad$ The standard for treatment of extremity soft tissue sarcomas is limbsparing surgery with a margin of $1-2 \mathrm{~cm}$. Overlying fascial layers (i.e. muscular fascia, femoral sheath, periosteum) are often barriers to tumor extension and are acceptable margins when major neurovascular or bony structures are in close proximity.

- $\quad$ Rates of local and distant recurrence vary by histologic subtype. These differences inform surgical margins as well as the use of chemotherapy and radiation.

- $\quad$ Radiation therapy is used to decrease rates of local recurrence in highrisk tumors. Neoadjuvant (vs. adjuvant) radiation can minimize side effects to nearby joints and normal tissues, but is associated with increased rates of wound complications and has equivalent rates of local control.

- Use of adjuvant chemotherapy is controversial. Neoadjuvant chemotherapy should be routinely prescribed for high-risk, chemosensitive subtypes (i.e. Ewing sarcoma and rhabdomyosarcoma). It can be selectively prescribed for moderately chemosensitive subtypes based on other risk factors such as size. 


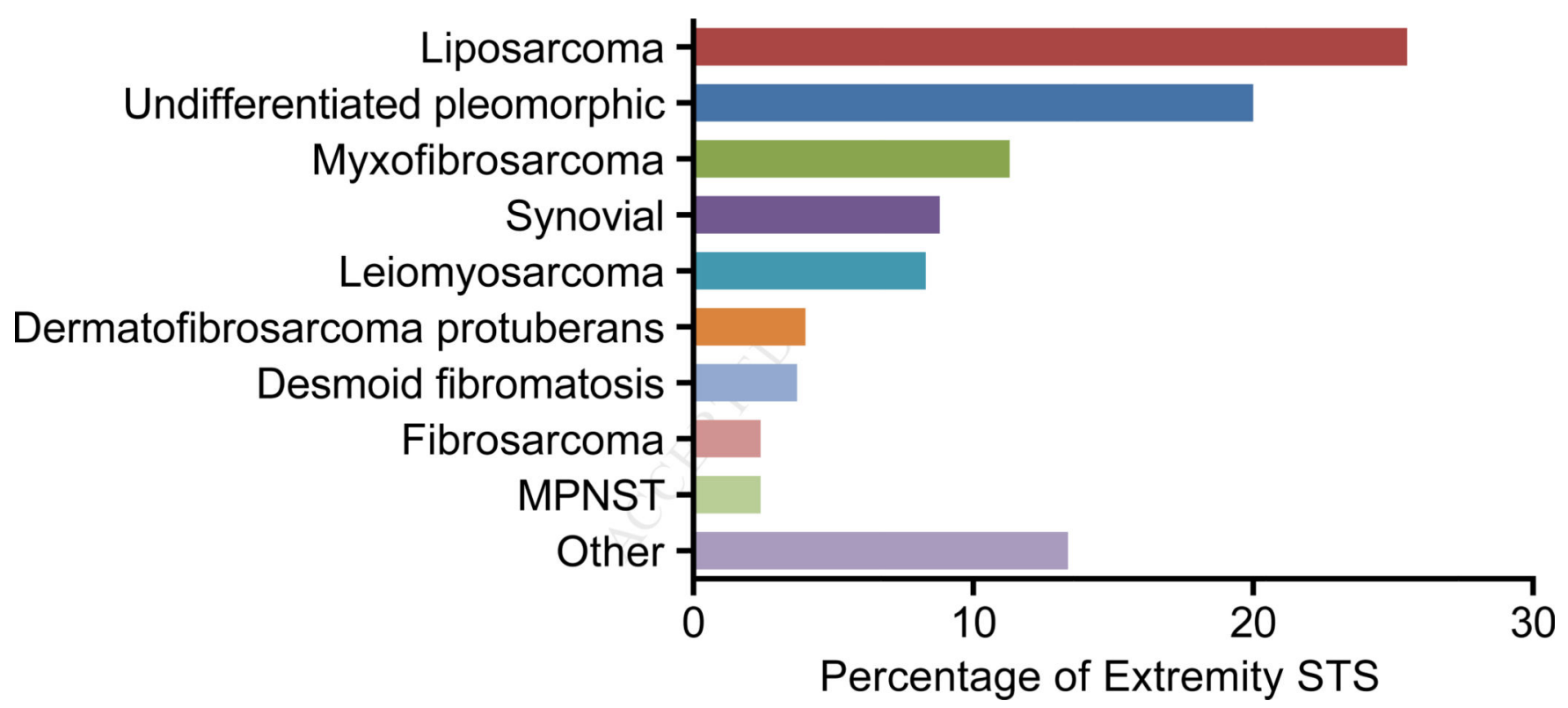

Figure 1.

Histologic distribution of primary extremity soft tissue sarcomas $(n=3,103)$. The data are derived from all surgically resected soft tissue sarcomas followed prospectively at Memorial Sloan-Kettering Cancer Center between 1980 and 2014. Tumors previously designated as malignant fibrous histiocytoma are denoted as undifferentiated pleomorphic sarcoma. Histologic subtypes that represented $\mathcal{2} \%$ of all cases are grouped as other. MPNST, malignant peripheral nerve sheath tumor. 

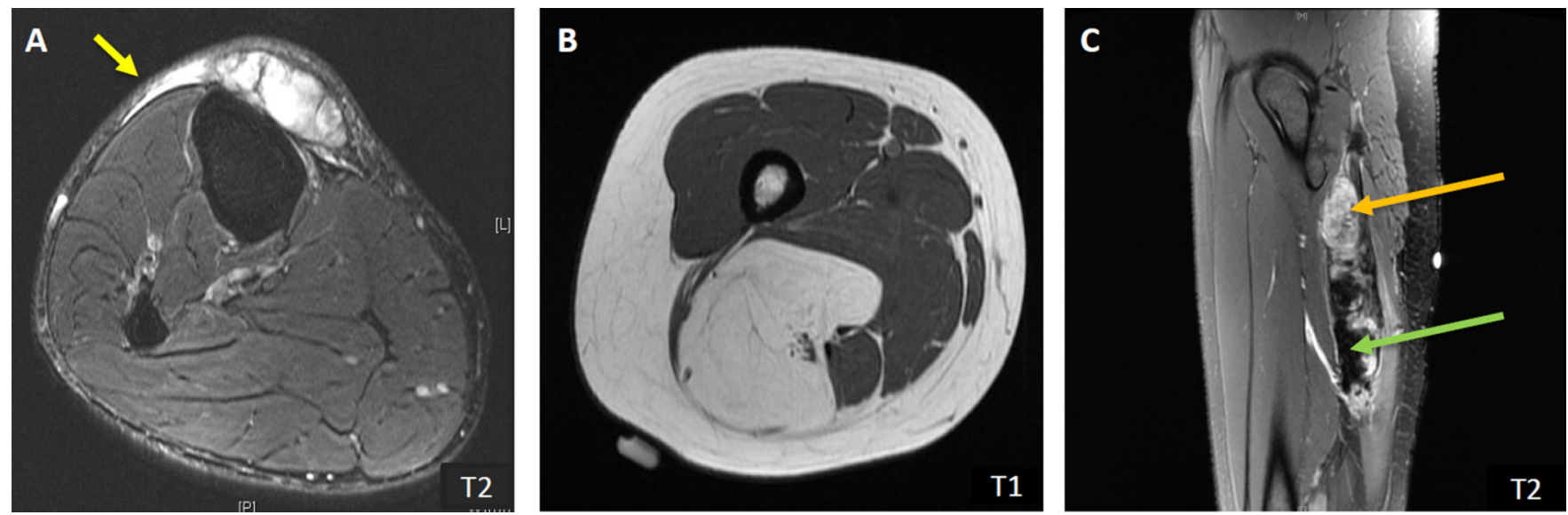

Figure 2.

A) A T2-enhancing tail (arrows) is often seen in myxofibrosarcoma, representing its infiltrative borders. B) A lipomatous mass in the posterior thigh compartment with multiple septations consistent with an atypical lipomatous tumor. C) Desmoids are mixed intensity on T2-weighted imaging, with high-T2 areas representing cellular components (orange arrow) and low-T2 areas representing collagenous components (green arrow). 
Local Recurrence-Free Survival

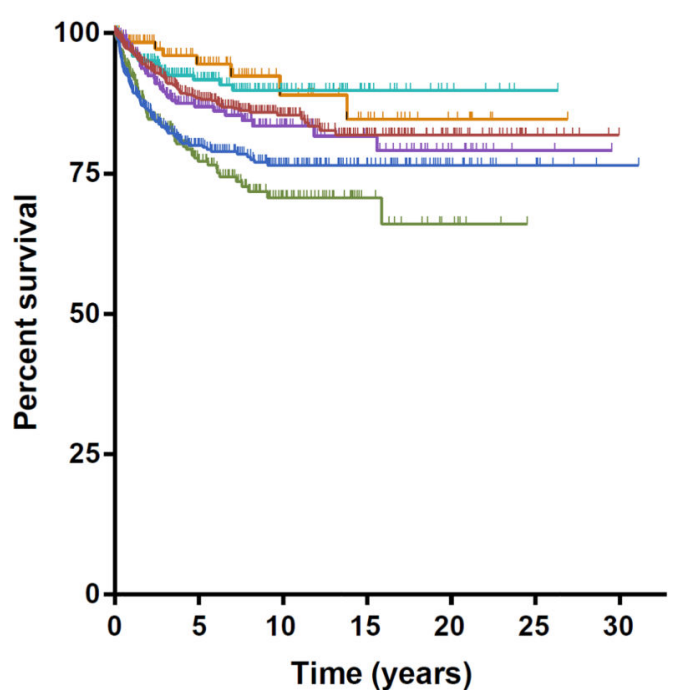

Distant recurrence-free survival

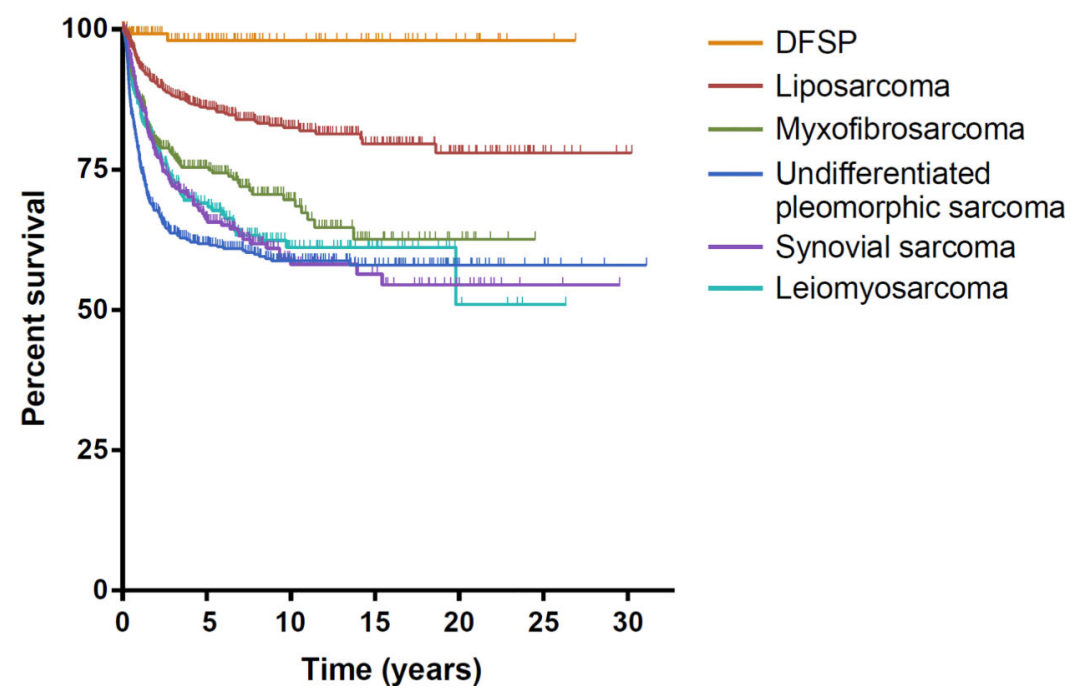

Figure 3.

Local recurrence-free survival and distant recurrence-free survival for the most common subtypes of extremity sarcoma $(n=2498)$. DFSP, dermatofibrosarcoma protuberans. 


\section{Table 1}

Characteristic genomic alterations and diagnostic testing for different extremity histologic subtypes

\begin{tabular}{lll}
\hline Histologic Subtype & Genomic Alteration & Diagnostic Testing \\
\hline Atypical lipomatous tumor and & $12 \mathrm{q} 13-15$ amplification & FISH or IHC for $C D K 4$ or \\
Dedifferentiated liposarcoma & & MDM2 \\
Desmoid fibromatosis & CTNNB1 mutation or APC loss & IHC for nuclear $\beta$-catenin \\
Epithelioid sarcoma & SMARCB1 deletion & IHC for SMARCB1 \\
Myxoid and Round cell liposarcomas & FUS-DDIT3 fusion or & FISH or PCR for FUS-DDIT3 \\
& EWSR 1-DDIT3 fusion & or EWSR 1-DDIT3 \\
Synovial sarcoma & $S Y T-S S X 1, S Y T-S S X 2$, or & PCR for SYT-SSX1-3 \\
& $S Y T-S S X 3$ fusion & PCR for EWSR1-FLI1 \\
Ewing's sarcoma & EWSR1-FLI1 fusion & PCR for COL1A1-PDGFB \\
Dermatofibrosarcoma protuberans & COL1A1-PDGRB fusion & IHC for STAT6 \\
Solitary fibrous tumor & NAB2-STAT6 fusion & \\
\hline
\end{tabular}




\section{Table 2}

AJCC staging for soft tissue sarcoma $\left(7^{\text {th }}\right.$ ed, 2010)

\begin{tabular}{l|l}
\hline Primary tumor $(\mathbf{T})$ \\
\hline TX & Primary tumor cannot be assessed \\
\hline T0 & No evidence of primary tumor \\
\hline T1 & Tumor $5 \mathrm{~cm}$ in greatest dimension \\
T1a & Superficial tumor (not involving underlying fascia) \\
T1b & Deep tumor (involving or deep to fascia) \\
\hline T2 & Tumor $>5 \mathrm{~cm}$ in greatest dimension \\
T2a & Superficial tumor \\
T2b & Deep tumor
\end{tabular}

\section{Regional lymph nodes (N)}

\begin{tabular}{l|l}
\hline NX & Regional lymph nodes cannot be assessed \\
\hline No & No regional lymph node metastasis \\
\hline N1 & Regional lymph node metastasis
\end{tabular}

Distant metastases (M)

\begin{tabular}{l|l}
\hline M0 & No distant metastasis \\
\hline M1 & Distant metastasis \\
\hline
\end{tabular}

Histologic Grade (G)

\begin{tabular}{l|l}
\hline $\mathbf{G X}$ & Grade cannot be assessed \\
\hline
\end{tabular}

G1 $\mid$ Grade 1

\begin{tabular}{l|l}
\hline G2 & Grade 2
\end{tabular}

\begin{tabular}{l|l}
\hline G3 & Grade 3 \\
\hline
\end{tabular}

\begin{tabular}{|l|c|c|c|c|}
\hline Stage & T & N & M & G \\
\hline Stage IA & T1a & N0 & M0 & G1, GX \\
& T1b & N0 & M0 & G1, GX \\
\hline Stage IB & T2a & N0 & M0 & G1, GX \\
& T2b & N0 & M0 & G1, GX \\
\hline Stage IIA & T1a & N0 & M0 & G2, G3 \\
& T1b & N0 & M0 & G2, G3 \\
\hline Stage IIB & T2a & N0 & M0 & G2 \\
& T2b & N0 & M0 & G2 \\
\hline Stage III & T2a & N0 & M0 & G3 \\
& T2b & N0 & M0 & G3 \\
& Any T & N1 & M0 & Any G \\
\hline Stage IV & Any T & Any N & M1 & Any G \\
\hline
\end{tabular}


Adapted from Edge SB, Byrd, DR, Compton CC, et al (eds): AJCC Cancer Staging Manual, $7^{\text {th }}$ ed. New York, Springer, 2010; with permission. 


\section{Table 3}

Principles of surgery in extremity soft tissue sarcoma

Core biopsy should be done in line with the planned surgical incision and then excised at the time of definitive resection Plan for 1-cm margin except for the infiltrative subtypes (myxofibrosarcoma and DFSP), which require a 2-cm margin.

Superficial sarcomas should be excised with the underlying fascia. For deep/intramuscular tumors, the fascia between muscle bodies or compartments provides a good barrier to tumor extension and should be resected with the specimen.

Skeletonize vessels and motor nerves unless encased by high-grade sarcoma. Low-grade tumors can be bivalved around critical structures to minimize morbidity 


\section{Table 4}

Advantages of neoadjuvant vs. adjuvant radiation therapy

\begin{tabular}{ll}
\hline Neoadjuvant & Adjuvant \\
\hline Decreased joint toxicity & Fewer wound complications \\
Decreased field size and radiation dose & \\
Decreased surgical field & \\
Avoid radiating complex reconstruction & \\
\hline
\end{tabular}


Table 5

Risk of distant metastases and chemosensitivity for high grade extremity sarcomas

\begin{tabular}{|c|c|l|l|l|}
\hline \multicolumn{2}{|c|}{} & \multicolumn{3}{c|}{ Chemosensitivity } \\
\cline { 3 - 5 } \multicolumn{2}{|c|}{} & \multicolumn{1}{|c|}{ Low } & Moderate & High \\
\hline \multirow{4}{*}{$\begin{array}{c}\text { Risk of Distant } \\
\text { Metastases }\end{array}$} & Lowerate & $\begin{array}{l}\text { Dedifferentiated } \\
\text { liposarcoma }\end{array}$ & Myxofibrosarcoma & \\
\cline { 2 - 5 } & High & & $\begin{array}{l}\text { Undifferentiated pleomorphic } \\
\text { sarcoma } \\
\text { Leiomyosarcoma }\end{array}$ & \\
\cline { 2 - 5 } & & $\begin{array}{l}\text { Round cell liposarcoma } \\
\text { Pleomorphic liposarcoma } \\
\text { Angiosarcoma } \\
\text { Synovial sarcoma }\end{array}$ & $\begin{array}{l}\text { Ewing sarcoma } \\
\text { Rhabdomyosarcoma }\end{array}$ \\
\hline
\end{tabular}

